\title{
An update of the classical Bokhman's dualistic model of endometrial cancer
}

\author{
Miłosz Wilczyński ${ }^{1}$, Justyna Danielska², Jacek Wilczyński ${ }^{3}$ \\ ${ }^{1}$ Polish Mother's Memorial Hospital Research Institute, Department of Operative Gynecology, Endoscopy and Gynecologic Oncology, \\ Lodz, Poland \\ ${ }^{2}$ Radiotherapy Department, Medical Univeristy of Lodz, Poland \\ ${ }^{3}$ Polish Mother's Memorial Hospital Research Institute, Department of Operative Gynecology and Gynecologic Oncology, Lodz, Poland
}

\begin{abstract}
According to the classical dualistic model introduced by Bokhman in 1983, endometrial cancer (EC) is divided into two basic types. The prototypical histological type for type I and type II of EC is endometrioid carcinoma and serous carcinoma, respectively. The traditional classification is based on clinical, endocrine and histopathological features, however, it sometimes does not reflect the full heterogeneity of EC. New molecular evidence, supported by clinical diversity of the cancer, indicates that the classical dualistic model is valid only to some extent. The review updates a mutational diversity of EC, introducing a new molecular classification of the tumour in regard to data presented by The Cancer Genome Atlas Research Network (TGCA).
\end{abstract}

Key words: endometrial carcinoma, genomics, review.

\section{Introduction}

According to the World Cancer Research Fund International, endometrial cancer $(\mathrm{EC})$ is the sixth most common women's malignancy worldwide [1]. It is estimated that $\mathrm{EC}$ is the second most frequent cancer of the female reproductive organs, just after cervical cancer. There were 320000 newly diagnosed cases in 2012 [2]. Due to the longer expected life duration, increase in obesity among women, EC has become the most commonly diagnosed tumour of female reproductive organs in developed countries. The incidence of endometrial cancer is going to increase in the upcoming years. If endometrial cancer is diagnosed in the early stage, chances of survival are high (even more than $75 \%$ patients with 5 -year survival for all EC cases, up to $90 \%$ in early EC) [3]. The highest incidence of EC is observed in menopausal women, especially between 55 and 60 years of age [4]. In 1983, Bokhman introduced a dualistic model of two pathogenetic types of EC in women [5]. After more than 30 years this classification seems to be valid, however, it requires an update based on new molecular discoveries and clinical insights.

\section{Bokhman's classification}

The traditional classification of epithelial tumours, which was proposed in Bokhman's publication, is based on clinical, metabolic and endocrine characteristics of
EC. The author postulated that there are two different pathogenetic types of EC (frequency: type I-70\%, type II - 30\%) [5]. The first type is present in obese woman afflicted by hyperlipidaemia / diabetes and is caused by hyperestrogenism due to anovulatory uterine bleeding, infertility or late onset of the menopause. Furthermore, type $I$ is associated with hormone receptor positivity and arises on the basis of endometrial hyperplasia. This type of EC is composed of moderately/highly differentiated tumours that are characterised by usually favourable prognosis [6-9]. In contrast, type II of EC is associated with atrophic endometrium, arises in nonobese women and is independent of metabolic or endocrine disturbances. This type of EC is composed of poorly differentiated tumours that are characterised by unfavourable prognosis. Type II neoplasms are clinically aggressive, have tendency to metastases and are diagnosed in the advanced stages of the disease. On the contrary, type I tumours are more likely to be diagnosed in the early stage [7-9]. It is hypothesised that type II cancers arise from a premalignant lesion - EIC (endometrial intraepithelial carcinoma), whereas type I tumours are associated with a distinct premalignant condition - EIN (endometrial intraepithelial neoplasia) $[10,11]$.

Since Bokhman's classification was introduced in 1983, molecular and histological studies have verified this hypothesis showing its clinical usefulness. EC in terms of histopathological characteristics is divided 
into several main and most frequent subtypes: serous carcinoma, clear-cell carcinoma, endometrioid adenocarcinoma and carcinosarcoma. Basing on the histopathological classification, type I tumours are usually low-grade endometrioid cancers, whereas type II are usually high-grade serous or clear-cell carcinomas. Molecular evidence shows discrete features of the two EC types in terms of gene copy numbers. Endometrioid carcinomas are characterised by mutations in PTEN, KRAS, CTNNB1, PIK3CA and microsatellite instability (MSI) [12-15]. Multiple studies devoted to the molecular basis of EC proved that endometrioid carcinomas are highly mutated tumours in regard to $\mathrm{PI} 3 \mathrm{~K} / \mathrm{AKT} / \mathrm{mTOR}$ and $W n t / \beta$-catenin signalling pathways [16]. PTEN is considered to be one of the most important negative regulators of the $\mathrm{PI} 3 \mathrm{~K} / \mathrm{AKT} / \mathrm{mTOR}$. LoSS of PTEN or its functions leads to the uncontrolled stimulation of the pathway and subsequent carcinogenesis. Dysfunction of tumour suppressor PTEN is present in the majority of endometrioid carcinomas. As PTEN loss is also frequently observed in endometrial hyperplasia, thus, it is hypothesised that it may be an initial and early step in the pathogenesis of endometrioid carcinoma [17, 18]. PIK3CA mutations are also associated with the disturbances in PI3K/AKT/mTOR pathway leading to deregulation of cell proliferation and apoptosis [16]. PIK3CA gene encodes catalytic subunit $p 110-\alpha$. When mutated, it leads to uncontrolled activation of Akt kinase. PIK3 deregulation is observed more frequently in endometrioid carcinomas [19]. Wnt/ $\beta$-catenin pathway is dysregulated in both endometrioid and serous carcinomas, however, mutations of CTNNB1 are observed almost only in endometrioid carcinomas. CTNNB1 gene encodes $\beta$-catenin, the key regulator enzyme of the whole pathway. Activation of the pathway leads to the accumulation of $\beta$-catenin and localization in the nucleus, which leads to upregulation of many target genes such as VEGF, Myc, cyclin D or E-cadherin [16]. On the contrary, non-endometrioid carcinomas (type II) are characterised by different molecular abnormalities showing distinct changes, such as HER2 amplification and TP53 mutations. TP53 gene encodes a tumour suppressor p53, which plays a crucial role in conserving stability in the human genome. TP53 mutations are present in up to $90 \%$ of serous carcinomas [20]. HER2 amplification is characteristic of type II of EC. It is an oncogene that is encoded by $E R B B 2$ gene and is a member of the human epidermal growth factor receptor (HER/EGFR/ERBB) family (Tables I, II) [21].

\section{Inconsistency in classical Bokhman's classification}

The Bokhman's dualistic model of EC seems to be very useful to divide tumours into two subtypes, which allows to predict possible prognosis and introduce proper treatment. What is more, the above-mentioned model was used as a basis for multiple molecular studies devoted to the pathogenesis of two types of EC. However, Bokhman's conception seems to be oversimplified, introducing two homogenous types of EC. Clinical, epidemiological, and molecular evidence suggests that dividing EC into just two types does not reflect the true nature of the endometrial malignant pathology. Furthermore, original Bokhman's classification did not include clear-cell carcinoma which is considered by many authors as a type II of EC. Such neoplasms as carcinosarcomas or other undifferentiated carcinomas are omitted in the classification [5]. Both types of EC seems to be more heterogeneous than it has been thought before. Furthermore, there is a subtype of tumours that share mutual features of endometrioid and

Tab. I. Distinct clinicopathological features of type I and type II endometrial cancer

\begin{tabular}{lcc}
\hline Parameter & Type I & Type II \\
\hline Histopathological type & endometrioid & serous, clear-cell \\
\hline Grading & G1-G2 & G3 \\
\hline Prognosis & favourable & poor \\
\hline Clinical course & non-aggressive & aggressive \\
\hline Clinical stage at diagnosis (FIGO) & I-II & III-IV \\
\hline Receptor positivity (oestrogens/progesterone) & + & - \\
\hline Oestrogen associated & + & - \\
\hline Endometrium & hyperplasia & atrophy \\
\hline Metastases & seldom & frequent \\
\hline Myometrial invasion & usually less than 1/2 of the uterine wall & deep \\
\hline Sensitivity to hormonal therapy & + & - \\
\hline
\end{tabular}


serous cancers. Such risk factors as obesity or diabetes are mainly associated with type I of EC, however, epidemiological data suggest that to some extent those factors are present in type II patients. It has to be brought to attention that the classical classification was based on clinical findings that were noticed in the second half of the $20^{\text {th }}$ century. Since then, obesity and other civilization diseases have spread throughout societies of developed countries contributing to the increase in the risk of many cancers, including endometrial neoplasms. Therefore, some patients with type II of EC present similar co-diseases as type I patients [22]. Low-grade endometrioid and serous carcinomas belong to the two types of EC that reflect distant extremes in regard to clinical and molecular characteristics ("pure" type I or II of EC). However, high-grade carcinomas may be an overlap between the two types, presenting many mutual features that are characteristic of both groups of cancer. Despite the fact that high-grade endometrioid carcinomas histologically are different from serous carcinomas, they present similar clinical and pathological characteristics [23]. They tend to form distant and nodal metastases, deeply infiltrate myometrium, spread aggressively and develop in a short period of time. All of these characteristics mentioned above cause poor prognosis in case of high-grade endometrioid carcinomas similarly to the serous counterparts. On the other hand, some serous carcinomas behave clinically as type I of EC. Up to $20 \%$ of serous cancers do not deeply infiltrate the myometrium, and a small number of them are associated with endometrial hyperplasia [24]. Discrimination between serous and high-grade endometrioid carcinomas may be difficult even for an experienced pathologist. Use of immunohistochemical markers is sometimes the only way to properly classify tumours.

Molecular data show the heterogeneity of endometrial malignancy and underline the fact that type I and type II of EC share similar characteristics. Despite the fact that both types of EC are characterised by distinct genetic alterations, there is a set of mutations that can be found in endometrioid and serous carcinomas as well [26]. TP53 mutations are typical of serous tumours, however, about $10 \%$ of endometrioid carcinomas also exhibit this molecular feature [12, 26]. Dysregulation of PIK3/Akt/mTOR pathway is also often found in type II of EC [26]. PIK3C may be observed even in $40 \%$ of cases, depending on the study. Similarly, loss of PTEN function may play an important role not only in endometrioid but also in serous cancers.

\section{New molecular classification - The Cancer Genome Atlas Research Network}

In 2013, TGCA (The Cancer Genome Atlas Research Network) presented the first large-scale, comprehen-
Tab. II. Distinct genetic alterations in type I and type II endometrial cancer (percentage is based on several publications, showing only a general tendency) [24-26]

\begin{tabular}{lcc}
\hline Mutation & Type I & Type II \\
\hline PTEN & $50-80 \%$ & $1-10 \%$ \\
\hline KRAS & $20-40 \%$ & $4-8 \%$ \\
\hline CTNNB1 & $25 \%$ & uncommon \\
\hline PIK3CA & $40-50 \%$ & $20-40 \%$ \\
\hline PIK3R1 & $20-40 \%$ & $<10 \%$ \\
\hline ARID1A & $25-50 \%$ & $5-10 \%$ \\
\hline TP53 & $10 \%$ & $80-90 \%$ \\
\hline HER2 & very uncommon & $30-40 \%$ \\
\hline MSI & $30-40 \%$ & uncommon \\
\hline PPP2R1A & $<10 \%$ & $10-40 \%$ \\
\hline
\end{tabular}

sive genomic characterisation of EC [26-29]. The study was based on 307 endometrioid, 57 serous and 13 mixed (serous/endometrioid) carcinomas. Next generation sequencing of the whole exome, genome-wide copy number analysis, whole transcriptome sequencing, methylation profiling, reverse phase protein array and assessment of microsatellite instability were used to evaluate the mutational landscape of EC. Results of the study allowed to categorise endometrial cancer into four distinct genomic subtypes, basing on the integration of somatic mutate rates, copy number alterations and microsatellite instability.

\section{POLE ultramutated subgroup}

This subgroup is characterised by an extremely high mutation rate $\left(232 \times 10^{-6}\right.$ mutations/Mb), which is associated with somatic alterations in the exonuclease domain of POLE. POLE encodes the central catalytic subunit of the polymerase epsilon which is involved in the DNA repair, correcting possible errors done during synthesis of DNA [30]. TGCA discovered 190 SMGs (significantly mutated genes) in the POLE ultramutated type, including mutations in PTEN, PIK3R1, PIK3CA and KRAS. PTEN alteration was detected in $94.1 \%$ of tumours. This subgroup was also characterised by a high rate of $\mathrm{C}>\mathrm{A}$ transversions, few copy number $\mathrm{ab}$ errations. In the study, $6.4 \%$ of low-grade and $17.4 \%$ of high-grade endometrioid carcinomas belonged to the ultramutated subgroup. None of serous or mixed ECs was noted. In comparison to other subgroups (hypermutated/MSI, copy number low/MSS, or copy number high/serous-like), ultramutated carcinomas were associated with a longer period of progression-free survival in patients. 
Hypermutated group, microsatellite instability (MSI)

This subgroup is characterised by a high mutation rate $\left(18 \times 10^{-6}\right.$ mutations/Mb), low level of somatic copy number alterations and is composed of microsatellite unstable tumours. The hypermutated subgroup presents a reduced $M L H 1$ gene expression due to the methylation of its promoter. In the TCGA study, 28.6\% of low-grade and $54.3 \%$ of high-grade endometrioid carcinomas belonged the hypermutated/MSI+ group. None of serous or mixed ECS was noted. MSI is infrequently found in serous ECS, which was proved by other authors. TGCA study identified 21 SMGs in the hypermutated/MSI+ group, including 11 genes (ARID5B, CSDE1, CTCF, GIGYF2, HIST1H2BD, LIMCH1, MIR1277, NKAP, RBMX, TNFAIP6, ZFHX3) that previously had never been connected with EC pathogenesis. However, mutations of genes that are commonly altered during endometrial carcinogenesis were also noticed (PTEN, PIK3CA, PIK3R1, CTNNB1, KRAS, ARID1A). PTEN mutations were found to be the most frequent in the subgroup and alterations of the PTEN-PIK3CA axis seemed to be common. KRAS alterations were detected in approximately $35 \%$ of tumours. CTNNB1 mutations were detected in approximately $20 \%$ of tumours, which was less frequent in comparison to the MSI stable tumours. Furthermore, recurrent RPL22 frameshift deletions were also observed, being characteristic of the hypermutated subgroup. Previous studies proved that RPL 22 mutations may occur more frequently in microsatellite highly instable endometrioid carcinomas [31]. Unfortunately, the exact function of RPL22 has yet to be determined. ARID5B mutations were found in about $23 \%$ of the MSI hypermutated subgroup. This finding was characteristic of the subgroup, being less frequent in ultramutated and MSI stable classes. ARID5B is a member of the human AT-rich interaction domain (ARID) family. ARID family members regulate transcription and take part in such processes as cell proliferation and differentiation, being active in cancer-related pathways.

\section{Copy number-low, microsatellite stable (MSS) subgroup}

This group is characterised by a lower mutation rate $\left(2.9 \times 10^{-6}\right.$ mutations/Mb) and is composed of the microsatellite stable endometrioid cancers. In TGCA study, $60 \%$ of low-grade and $8.7 \%$ of high-grade endometrioid carcinomas belonged to the MSS/copy number-low group. Additionally, 25\% of mixed and $2.3 \%$ of serous cancers were also found in this subgroup. However, only grade $\mathrm{G} 1$ and $\mathrm{G} 2$ endometrioid cancers' rate was significantly high. TGCA study identified 16 SMGs in the MSS/copy number-low subgroup, including seven genes (CSMD3, CTCF, BCOR, SOX17, MECOM, METTL14, SGK1) that had never been linked with endometrial carcino- genesis before. Apart from them, nine commonly known genes in regard to EC were identified (PTEN, PIK3CA, PIK3R1, ARID1A, CTNNB1, SPOP, CHD4, KRAS, FGFR3). CTNNB1 mutations, which dysregulate the Wnt/ $\beta$-catenin pathway, were considerably common $-52 \%$. The SOX17 gene, which regulates $\beta$-catenin expression, was altered only in this subgroup. Due to the somatic mutations, PIK3 pathway was dysregulated in more than $90 \%$ of tumours. Microsatellite stable tumours are usually characterised by low rates of KRAS mutations. KRAS was altered in about $16 \%$ of cases. This finding is in line with previous studies by other authors, which showed a higher KRAS mutation rate of MSI unstable cancers [32].

Expression of the progesterone receptor was elevated in the copy number-low subgroup, which allows to predict hormonal responsiveness of these tumours.

\section{Copy number-high, serous-like tumours}

This group consists of almost all serous carcinomas (97.7\%), $19.6 \%$ of high-grade endometrioid carcinomas, $5 \%$ of low-grade endometrioid carcinomas and $75 \%$ of mixed histology tumours. The copy number-high subgroup is characterised by a low mutation rate $(2.3 \times$ $10^{-6}$ mutations/Mb) and extensive copy number aberrations. Serous-like tumours were associated with poorer prognosis in comparison to other subgroups. TGCA described eight SMGs in this group, with TP53 mutation being the most common (91.7\%). Genetic instability and TP53 alterations have been previously suggested by other authors to be crucial in serous endometrial carcinomas' pathogenesis. Other SMGs described by TGCA also play an important role in the carcinogenesis. Alterations of such genes as PIK3CA, PIK3R1, PPP2R1A were identified, which were described as common in uterine serous tumours in several previous studies. Furthermore, recurrent FBXW7 and CHD4 mutations were detected. PIK3CA-PIK31R-PTEN axis is disturbed in 73\% of serous-like tumours. However, PTEN mutations is an infrequent event in this group. Similarly, KRAS alterations affect less than $10 \%$ of tumours in the subgroup. TGCA study revealed that $25 \%$ of the serous-like tumours were ERBB2-amplified.

\section{Conclusions from TGCA study}

It has to be brought to attention that approximately one fifth of endometrioid carcinomas were classified as serous-like tumours by TGCA's molecular studies. This fact discredits the classical Bokhman's classification, which divides EC just into two types. A subset of patients with high-grade endometrioid cancers may benefit from more aggressive treatment that is usually preferred in serous carcinomas. TGCA study may serve 
Tab. III. Overview of the TGCA subgroups [26-29]

\begin{tabular}{|c|c|c|c|c|}
\hline Parameter & POLE ultramutated & MSI+/hypermutated & Copy number low/MSS & Copy number high/serous-like \\
\hline Mutation rate & $232 \times 10^{-6} / \mathrm{Mb}$ & $18 \times 10^{-6} / \mathrm{Mb}$ & $2.9 \times 10^{-6} / \mathrm{Mb}$ & $2.3 \times 10^{-6} / \mathrm{Mb}$ \\
\hline Histological type & endometrioid & endometrioid & mainly endometrioid & endometrioid, serous and mixed \\
\hline Grade & G1-G3 & G1-G3 & predominance of G1-G2 & G3 \\
\hline Survival & favourable & intermediate & intermediate & poor \\
\hline $\begin{array}{l}\text { Mutated genes - } \\
\text { most important } \\
\text { examples (\%) }\end{array}$ & $\begin{array}{c}\text { POLE }(100 \%) \\
\text { PTEN (94.1\%) } \\
\text { MUC16 }(94.1 \%) \\
\text { RGDP13 }(94.1 \%) \\
\text { PIK3CA }(71 \%) \\
\text { PIK3RI }(65 \%) \\
\text { KRAS }(53 \%) \\
\text { ARID1A }(76 \%) \\
\text { GPR112 }(94.1 \%) \\
\text { CCNB3 }(94.1 \%) \\
\text { SLITRK3 }(94.1 \%) \\
\text { DNAH5 }(94.1 \%) \\
\text { APOB }(94.1 \%) \\
\text { LAMA2 }(94.1 \%) \\
\text { NBEA }(94.1 \%)\end{array}$ & $\begin{array}{l}\text { PTEN }(87.7 \%) \\
\text { PIK3CA }(53.8 \%) \\
\text { PIK3R1 }(41.5 \%) \\
\text { ARID1A }(36.9 \%) \\
\text { RPL22 }(36.9 \%) \\
\text { KRAS }(35.4 \%) \\
\text { ZFHX3 }(30.8 \%) \\
\text { ARID5B }(23.1 \%) \\
\text { CTNNB1 }(20 \%) \\
\text { FGFR2 }(13.8 \%)\end{array}$ & $\begin{array}{c}\text { PTEN }(76.7 \%) \\
\text { PIK3CA }(53.3 \%) \\
\text { CTNNB1 }(52.2 \%) \\
\text { ARID1A }(42.2 \%) \\
\text { PIK3R1 }(33.3 \%) \\
\text { CTFC }(21.1 \%) \\
\text { KRAS }(15.6 \%) \\
\text { FGFR2 }(13.3 \%)\end{array}$ & $\begin{array}{c}\text { TP53 }(91.7 \%) \\
\text { PIK3CA }(46.7 \%) \\
\text { FBXW7 }(21.7 \%) \\
\text { PPP2R1A }(21.7 \%) \\
\text { PIK3R1 }(13.3 \%) \\
\text { CHD4 }(13.3 \%) \\
\text { PTEN }(10.0 \%)\end{array}$ \\
\hline
\end{tabular}

as a basis for a new molecular classification, which may complement or even replace the classical dualistic model proposed by Bokhman [33].

POLE hotspot mutations were identified exclusively in the first genomic group, which consists of ultramutated tumours. POLE alterations may be a promising biomarker that may indicate favourable prognosis in patients [33].

PIK3 pathway was altered in majority of MSI+/hypermutated and copy number-low endometrioid cancers and affected more than 50\% of serous-like/copy number-high cancers. PTEN mutations were observed in almost $90 \%$ of all endometrioid cancers included in the study (Table III) [26].

\section{Disclosure}

Authors report no conflict of interest.

\section{References}

1. World Research Cancer Fund International: www.wcrf.org

2. Ferlay J, Soerjomataram I, Ervik M, et al. Cancer Incidence and Mortality Worldwide: IARC CancerBase No. 11. GLOBOCAN 2012 v1.1, France: International Agency for Research on Cancer; 2014. www.globocan.iarc.fr.

3. National Cancer Institute: www.cancer.gov.p

4. Krajowy Rejestr Nowotworów: www.onkologia.org.pl

5. Bokhman JV. Two pathogenetic types of endometrial carcinoma. Gynecol Oncol 1983; 15: 10-17.

6. Sorosky JI. Endometrial Cancer. Obstet Gynecol 2012; 120: 383-397.

7. Fisher B, Costantino JP, Redmond CK, et al. Endometrial cancer in tamoxifen-treated breast cancer patients: findings from the National Surgical Adjuvant Breast and Bowel Project (NSABP) B-14. J Natl Cancer Inst 1994; 86: 527-537.

8. Renehan AG, Tyson M, Egger M, et al. Body-mass index and incidence of cancer: a systematic review and meta-analysis of prospective observational studies. Lancet 2008; 371: 569-578.
9. McPherson CP, Sellers TA, Potter JD, et al. Reproductive factors and risk of endometrial cancer. The lowa Women's Health Study. Am J Epidemiol 1996; 143: 1195-1202.

10. Hecht JL, Ince TA, Baak JP, et al. Prediction of endometrial carcinoma by subjective endometrial intraepithelial neoplasia diagnosis. Mod Pathol 2005; 18: 324-330.

11. Mutter GL, Baak JP, Crum CP, et al. Endometrial precancer diagnosis by histopathology, clonal analysis, and computerized morphometry. J Pathol 2000; 190: 462-469.

12. McConechy MK, Ding J, Cheang MC, et al. Use of mutation profiles to refine the classification of endometrial carcinomas. J Pathol 2012; 228: 20-30.

13. Dedes KJ, Wetterskog D, Ashworth A, et al. Emerging therapeutic targets in endometrial cancer. Nat Rev Clin Oncol 2011; 8: 261-271.

14. Matias-Guiu X, Prat J. Molecular pathology of endometrial carcinoma. Histopathology 2013; 62: 111-123.

15. Salvesen HB, Haldorsen IS, Trovik J. Markers for individualised therapy in endometrial carcinoma. Lancet Oncol 2012; 13: e353-e361.

16. Markowska A, Pawałowska M, Lubin J, Markowska J. Signalling pathways in endometrial cancer. Contemp Oncol (Pozn) 2014; 18: 143-148.

17. Mutter GL, Lin MC, Fitzgerald JT, et al. Altered PTEN expression as a diagnostic marker for the earliest endometrial precancers. J Natl Cancer Inst 2000; 92: 9241-9230.

18. Oda K, Stokoe D, Taketani Y, McCormick F. High frequency of coexistent mutations of PIK3CA and PTEN genes in endometrial carcinoma. Cancer Res 2005; 65: 10669-10673.

19. Hiles ID, Otsu M, Volinia S, et al. Phosphatidylinositol 3-kinase: structure and expression of the 110 kd catalytic subunit. Cell 1992; 70: 419-429.

20. Prat J, Gallardo A, Cuatrecasas M, Catasus L. Endometrial carcinoma: pathology and genetics. Pathology 2007; 39: 1-7.

21. Buza N, Roque DM, Santin AD. Her2/neu in endometrial cancer: a promising therapeutic taret with diagnostic challenges. Arch Path Lab Med 2014; 138: 343-350.

22. Setiawan VW, Yang HP, Pike MC, et al. The Australian National Endometrial Cancer Study Group. Type I and II endometrial cancers: have they different risk factors? J Clin Oncol 2013; 31: 2607-2618.

23. Brinton LA, Felix AS, McMeekin DS, et al. Etiologic heterogeneity in endometrial cancer: evidence from a Gynecologic Oncology Group trial. Gynecol Oncol 2013; 129: 277-284.

24. Soslow RA, Bissonnette JP, Wilton A, et al. Clinicopathologic analysis of 187 high-grade endometrial carcinomas of different histologic subtypes: similar outcomes belie distinctive biologic differences. Am J Surg Pathol 2007; 31: 979-987. 
25. Lax SF. Molecular genetic pathways in various types of endometrial carcinoma: from a phenotypical to a molecular-based classification. Virchows Arch 2004; 444: 213-223.

26. Kandoth C, Schultz N, Cherniack AD, et al. The Cancer Genome Atlas Research Network. Integrated genomic characterization of endometrial carcinoma. Nature 2013; 497: 67-73.

27. Murali R, Soslow RA, Weigelt B. Classifi cation of endometrial carcinoma: more than two types. Lancet Oncol 2014; 15: e268-e278.

28. Le Gallo M, Bell DW. The Emerging genomic landscape of endometrial cancer. Clin Chem 2014; 60: 98-110.

29. Hong B, Le Gallo M, Bell DW. The mutational landscape of endometrial cancer. Curr Opin Genet Dev 2015; 30: 25-31.

30. Rohlin A, Zagoras T, Nilsson S, et al. A mutation in POLE predisposing to a multi-tumour phenotype. Int J Oncol 2014; 45: 77-81.

31. Novetsky AP, Zighelboim I, Thompson DM Jr, et al. Frequent mutations in the RPL22 gene and its clinical and functional implications. Gynecol Oncol 2013; 128: 470-474.

32. Byron SA, Gartside M, Powell MA, et al. Fgfr2 point mutations in 466 endometrioid endometrial tumors: Relationship with MSI, KRAS, PIK3CA, CTNNB1mutations and clinicopathological features. PLoS One 2012; 7: e30801.

33. Suh DH, Kim JW, Kang S, et al. Major clinical research advances in gynecologic cancer in 2013. J Gynecol Oncol 2014; 25: 236-248. 